\title{
Arbitrage and Control Problems in Finance. Presentation.
}

\author{
Elyès Jouini* \\ CEREMADE, Université de Paris-Dauphine \\ Place du Maréchal de Lattre de Tassigny \\ 75775 Paris Cedex, FRANCE
}

December 1, 2000

\section{Introduction}

The theory of asset pricing takes its roots in the Arrow-Debreu model (see, for instance, Debreu 1959, Chap. 7), the Black and Scholes (1973) formula, and the Cox and Ross (1976) linear pricing model. This theory and its link to arbitrage has been formalized in a general framework by Harrison and Kreps (1979), Harrison and Pliska (1981, 1983), and Duffie and Huang (1986). In these models, security markets are assumed to be frictionless: securities can be sold short in unlimited amounts, the borrowing and lending rates are equal, and there is no transaction cost. The main result is that the price process of traded securities is arbitrage free if and only if there exists some equivalent probability measure that transforms it into a martingale, when normalized by the numeraire. Contingent claims can then be priced by taking the expected value of their (normalized) payoff with respect to any equivalent martingale measure. If this value is unique, the claim is said to be priced by arbitrage and it can be perfectly hedged (i.e. duplicated) by dynamic trading. When the markets are dynamically complete, there is only one such a martingaleprobability measure and any contingent claim is priced by arbitrage. The weight of each state of the world for this probability measure can be interpreted as the state price of the economy (the prices of $\$ 1$ tomorrow in that state of the world) as well as the marginal utilities (for consumption in that state of the world) of rational agents maximizing their expected utility.

When there are frictions, including dynamic market incompleteness, the characterization of the no-arbitrage condition is nomore equivalent to the existence of a unique equivalent martingale measure. More precisely, for each

\footnotetext{
*Elyès Jouini is also affiliated with CREST, CEPR and Ecole Polytechnique de Tunisie. He wrote this presentation when he was visiting the finance department of the Stern School of Business, New York University.
} 
kind of imperfection, the equivalent martingale-measure condition is replaced by a weaker one : equivalent supermartingale-measure condition, equivalent submartingale-measure condition, absolutely continuous martingale-measure condition, etc. Besides, we generally have, more than one measure satisfying these conditions. Furthermore, when there are frictions, even if a contingent claim can be duplicated by dynamic trading, it is not necessarily possible to price it by arbitrage. However arbitrage bounds can be computed, for arbitrary contingent claims, taking the expected value of their (normalized) payoff with respect to all the measures that characterize the absence of arbitrage opportunities. These bounds are the minimum amount it costs to hedge the claim and the maximum amount that can be borrowed against it using dynamic strategies. These are the tightest bounds that can be inferred on the price of a contingent claim without knowing the agent's preferences. The determination of these bounds in a dynamic setting leads to a maximization (and/or minimization) program, and, in a dynamic setting, is often transformed into a stochastic optimal control problem. The main assumption in these models is, in fact, a necessary condition for the existence of an equilibrium: the no-arbitrage condition. These preference-free theories give results of great generality without specifying the equilibrium in its full details.

Another important class of valuation theories makes assumptions on preferences and derives more specific pricing restrictions than the preference-free theory does, even in the presence of imperfections. The price of a given contingent claim, for these theories, is just the expected value of its (normalized) terminal payoff with respect to a probability measure, whose density is proportional to the marginal utility (for consumption) of the considered agent. From a mathematical point of view, starting with a given utility function, the problem is to write the first order conditions of the agent's utility maximization program, taking into account the potential imperfections in the description of the budget constraints and/or of the strategies in order to characterize the marginal utility for consumption at the final date. In a multi-period setting, this maximization problem is a stochastic optimal control problem. The main advantage of this approach is that it leads to a unique price for a given contingent claim. The main drawback is that this price depends on the choice of the utility function and on the agent endowment. If the utility function belongs to a given connected class of utility functions, we will obtain an interval of possible prices for that claim. More specifically, if the considered class is the set of all von NeumannMorgenstern (VNM) increasing and concave utility functions, the set of possible prices is exactly the set obtained with the arbitrage approach as shown by Jouini and Kallal (1999). The unique way to obtain tighter bounds with the utilitymaximization approach seems then to consider specific functions, or specific sets of functions, smaller than the set of all the VNM ones.

In fact, there is an interesting link between the two approaches. Since the arbitrage upper bound for a given contingent claim is equal to the minimum amount it costs to hedge it, taking the market frictions into account, the agent's problem (maximization of the utility provided by the terminal payoff among the strategies satisfying a given dynamic budget constraint) can be transformed 
into a static problem where we maximize the utility among the set of contingent claims satisfying a budget constraint where the classical price functional is replaced by the arbitrage upper bound functional. If we have, for instance, an explicit formula for the arbitrage upper bound, it suffices then to solve a static maximization problem instead of the initial stochastic dynamic control problem. The characterization of the no-arbitrage assumption is therefore crucial in order to solve the contingent claim pricing problem as well as to solve the individual utility maximization problem of each agent in the economy. The last step, if we want to explore all the implications of the Arrow-Debreu model in this financial setting is then to write the equilibrium conditions in order to ensure that all the individual solutions are "compatible".

\section{The no-arbitrage condition}

Let $(\Omega, \mathcal{F}, \mathrm{P})$ be a probability space and $\left(\mathcal{F}_{t}\right)_{t \geq 0}$ be a filtration which models our information structure. This filtration is supposed to satisfy the usual conditions, i.e. the filtration is right continuous and $\mathcal{F}_{0}$ contains all negligible sets (if $B \subset A \in \mathcal{F}$ and $\mathrm{P}(A)=0$ then $\left.B \in \mathcal{F}_{0}\right)$. We also suppose that the sigmaalgebra $\mathcal{F}=\bigcup_{t>0} \mathcal{F}_{t}$, and we consider a real valued semimartingale $S$, which models the price process for the marketed claims. In the next, we will denote by $\mathbb{R}$ real line and by $\mathbb{R}_{+}$the set of nonnegative ones. Let us define, as in Delbaen and Schachermayer (1994), an admissible strategy as follows:

Definition 1 Let a be a positive real number. An $S$-integrable predictable process $H$, is called a-admissible if $H(0)=0$, and $(H \cdot S) \geq-a$ (i.e. for all $t \in \mathbb{R}^{+}$, the stochastic integral $(H \cdot S)_{t} \geq-$ a almost everywhere). $H$ is called admissible if it is admissible for some $a \in \mathbb{R}^{+}$.

This admissibility condition can be interpreted as a bounded losses condition for strategies with a zero initial investment.

We consider, as in Stricker (1990), the convex cone $K_{0}$ in the space $L_{0}$ of equivalent classes of measurable functions, defined up to equality almost everywhere, given by

$$
K_{0}=\left\{(H \cdot S)_{\infty} \mid H \text { admissible and }(H \cdot S)_{\infty}=\lim _{t \rightarrow \infty}(H \cdot S)_{t} \text { exists a.s. }\right\} .
$$

The set $K_{0}$ is then the set of all terminal payoffs obtained through some admissible strategy.

Definition 2 We say that the semi-martingale $S$ satisfies the condition of noarbitrage, $(N A)$, if $K_{0} \cap L_{0}^{+}=\{0\}$

Since $K_{0}$ represents the set of all admissible terminal payoffs the no-arbitrage condition amounts to say that it is impossible to obtain a non-negative, non-zero payoffs with a zero initial investment.

Assume that it is possible to separate $K_{0}$ and $L_{0}^{+}$in the sense that there exists a non-zero linear functional $f$ and a real number $c$ such that $f\left(K_{0}\right) \leq c$ and 
$f\left(L_{0}^{+}\right)>c$ and assume that the separating functional $f$ admits a representation as an expectation operator with respect to a probability measure Q, then under the (NA) condition, $\mathrm{Q}$ is equivalent to $\mathrm{P}$ and $\mathrm{E}_{Q}[f] \leq 0$ for each $f$ in $K_{0}$. In particular, for each $s<t, B \in F_{s}, \alpha \in \mathbb{R}$, we have $\alpha\left(S_{t}-S_{s}\right) 1_{B} \in K_{0}$, therefore, $\mathrm{E}_{Q}\left[\left(S_{t}-S_{s}\right) 1_{B}\right]=0$, and Q is a martingale measure for $S$.

Unfortunately, the (NA) condition is seldom sufficient to apply a separation theorem. In the case where $S$ is locally bounded we have the following:

Theorem 1 (Delbaen and Schachermayer, 1994) Let $S$ be a locally bounded real valued semi-martingale. There is an equivalent local martingale measure $Q$

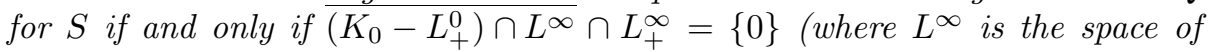
bounded measurable functions and where the closure is taken with respect to the norm-topology of $\left.L^{\infty}\right)$.

This last condition is called No Free-Lunch with Vanishing Risk (NFLVR) and deals with sequences of strategies such that the negative parts of their terminal payoff tends to zero uniformly instead of strategies with non-negative terminal payoff as in the (NA) condition. Remark that the main difference between these two conditions lies in the fact that we have to consider a closure. Indeed, the condition $\left[\left(K_{0}-L_{+}^{0}\right) \cap L^{\infty}\right] \cap L_{+}^{\infty}=\{0\}$ is weaker than $\left(K_{0}-L_{+}^{0}\right) \cap$ $L_{+}^{0}=\{0\}$, which is equivalent to the (NA) condition $K_{0} \cap L_{+}^{0}=\{0\}$.

If, in the previous condition, we replace the norm-topology closure by the $\sigma\left(L^{\infty}, L^{1}\right)$ topology closure (where $L^{1}$ is the space of all integrable $\mathcal{F}$-measurable functions) we obtain a version of the No Free-Lunch (NFL) condition introduced by Kreps (1981), and the existence of an equivalent local martingale measure is obtained for a bounded càdlàg, and adapted process $S$.

Other intermediary concepts, as the No Free-Lunch with Bounded Risk (NFLBR) condition (where the closure is defined as the set of weak*-limits and where the negative parts of the terminal payoffs tend to zero in probability and remains uniformly bounded), have been introduced in the literature and permit to obtain results similar to the previous theorem in different contexts: finite time set and (NA) condition with Dalang et al. (1989) (see Schachermayer (1992), Kabanov and Kramkov (1994a) and Rogers (1995) for elementary proofs), infinite but discrete time set with Schachermayer (1994), continuous and bounded processes in continuous time with Delbaen (1992). Harrison and Kreps (1979) and Harrison and Pliska (1981) used the concept of simple strategies. Kreps (1981) used a concept of "no free-lunch" involving the convergence of nets or generalised sequences. Duffie and Huang (1986) and Stricker (1990) used $L^{p}$ convergence. Lakner (1993) used convergence in Orlicz spaces. Furthermore, it appears that the class of semi-martingales is the most general one compatible with this kind of results. Indeed, from the work of Föllmer and Schweizer (1991) and Ansel and Stricker (1993) we know that No Free-Lunch conditions imply, in some sense, that $S$ is a semi-martingale. Conversely, the existence of an equivalent-martingale measure for $S$ implies, by Girsanov's theorem, that $S$ is a semi-martingale.

As in Harrison and Kreps (1979) and Kreps (1981) and for a given contingent claim, we define the arbitrage pricing interval as the set of all the prices that are 
compatible with the No Free-Lunch condition, i.e. introducing this contingent claim at one of these prices does not create free-lunches. In all the mentioned papers, it appears that this set is equal to the set of expected values of the considered claim terminal payoff with respect to all the probability-measures which characterize the absence of free-lunch.

When there is no imperfection, the cash-flow space $\Phi$ can be identified with $K_{0}$. In case of short-sale constraints, it can be identified with

$$
\left\{(H \cdot S)_{\infty} \mid H \text { admissible, } H \geq 0 \text { and }(H \cdot S)_{\infty}=\lim _{t \rightarrow \infty}(H \cdot S)_{t} \text { exists a.s. }\right\}
$$

and when there are short-selling costs, it can be identified with

$$
\left\{\begin{array}{l|l}
\left(H \cdot S-H^{\prime} \cdot S^{\prime}\right)_{\infty} \mid \begin{array}{l}
\left(H, H^{\prime}\right) \text { admissible, }\left(H, H^{\prime}\right) \geq 0 \\
\text { and }\left(H \cdot S-H^{\prime} \cdot S^{\prime}\right)_{\infty} \text { exists a.s. }
\end{array}
\end{array}\right\}
$$

where $S$ (resp. $S^{\prime}$ ) models the long (resp. short) position returns. When there are transaction costs, $\Phi$ can be identified with

$$
\left\{(H \cdot S)_{\infty}-\lambda V((H \cdot S)) \mid H \text { admissible, and }(H \cdot S)_{\infty} \text { exists a.s. }\right\}
$$

where $V$ represents the total variation of $(H \cdot S)$ and $\lambda$ the magnitude of the transaction costs. Jouini and Kallal (1995a and b) characterized first the absence of arbitrage opportunities in these different situations. Other contributions on related subjects are due to Kabanov and Kramkov (1994b), Shirakawa and Konno (1995), Kusuoka (1995), Cvitanic̀ and Karatzas (1996), Cvitanic̀, Pham and Touzi (1999), Kabanov (1999). The differences between all these references are in the choice of the topology (or no topology) in order to define the concept of free-lunch, the choice of a space of admissible strategies (discrete strategies, simple strategies,...) and finally the choice of possible imperfections (or no imperfection). This choice is summarized by the choice of a convex cone contained in $K_{0}-L_{+}^{0}$ instead of $K_{0}-L_{+}^{0}$ itself in order to model the opportunity set. In this context, it is easier to find a separating hyperplane between that set (or its closure with respect to some topology) and $L_{+}^{\infty}$. Jouini and Kallal (1999) extended all the arbitrage, viability and equilibrium classical results to that setting mainly by assuming that the opportunity set is a convex cone (or even a convex set) and the pricing rule is sublinear. In this issue, Kabanov and Stricker (2001) propose a generalization of Jouini and Kallal's (1995a) result to the important case of a multi-asset market model where the transaction costs are defined for each kind of transaction between any pair of assets. They use the geometric formalism developed previously by Kabanov (1999) and they characterize the absence of arbitrage opportunities in terms of martingale-like measures. Their result is established in a discrete time and finite set of states of the world framework and they only deal with arbitrages and not with freelunches.

In order to take a large set of possible frictions into account Carassus and Jouini $(1997,1998,2000)$ in discrete time or in a deterministic setting, Jouini and Napp (2000) and Jouini, Napp and Schachermayer (2000) in continuous 
time propose to deal directly with the space of possible cash-flows instead of the space of terminal payoffs and they provide a characterization of the No FreeLunch assumption in terms of the existence of a separating functional. Napp (2001) develops an arbitrage pricing theory and a super-replication concept in this cash-flow space.

However, all these results are obtained under a convexity condition on the space of attainable payoffs. This last assumption is not satisfied in economies with fixed costs, i.e. with transaction costs which are not proportional to the size of the transactions. In this framework, the terminal payoff of a strategy $H$ is $(H \cdot S)_{\infty}+c(H)$, where $c$ is a bounded non-linear function of the strategy $H$, instead of $(H \cdot S)_{\infty}$ as in the classical case. Therefore, it is easy to understand that large scale transactions will kill the transaction cost effect and that the characterization of the no-arbitrage condition should be an asymptotic version of the classical one. Jouini, Kallal and Napp (2001) prove that this characterization is in terms of absolutely continuous martingale measures and show that the existence of such a measure is necessary but not sufficient and that we need the existence of a family of such measures each one associated with a given date and a given event at that date in order to characterize the absence of free-lunches.

\section{The utility maximization problem}

Before Merton's (1969) paper, most models of portfolio selection only considered one period. Furthermore, the investment decision by households was viewed in two parts : (a) the "consumption-saving" choice where the individual decides how much income and wealth to allocate into current consumption and how much to save for future consumption; and (b) the "portfolio-selection" choice where the investor decides how to allocate savings among the available investment opportunities. Merton (1969) examined "the combined problem of optimal portfolio selection and consumption rules for an individual in a continuous-time model where his income is generated by returns on assets and these returns or instantaneous 'growth rates' are stochastic".

The original analysis of Merton's model is based on the Hamilton-JacobiBellman equation and requires an underlying Markov state process. After the papers of Harrison and Kreps (1979) and Harrison and Pliska (1981, 1983), and their characterization of the no-arbitrage assumption in terms of the existence of martingale-measures, Pliska (1986), Cox and Huang $(1989,1991)$ and Karatzas, Lehoczky and Shreve (1987) used this methodology in order to analyze this consumption-investment problem. This new approach is based on duality arguments and permits to transform the initial dynamic problem into a static one and to solve it without assuming any "Markov" condition.

Let us now introduce the main results related to this problem.

Let $(\Omega, F, P)$ be a fixed probability space and $\mathbf{T}$ denote the interval $[0, T]$, on which we are going to treat our problem : $T$ corresponds to the terminal date for all economic activity under consideration. All processes that we shall encounter in this section are defined on $\mathbf{T}$. 
We consider a market consisting in one bond and $N$ assets. More precisely, the primitive market model is the same as in Karatzas (1989), except that we consider here dividends paying assets.

We adopt a model for the market consisting of one bond with price at time $t$ denoted by $S_{t}^{0}$ satisfying the differential equations

$$
d S_{t}^{0}=S_{t}^{0} r_{t} d t, \quad S_{0}^{0}=1,
$$

and $N$ stocks with prices at time $t$ denoted by the $N$-dimensional vector ${ }^{1} S_{t}$ satisfying

$$
d S_{t}=S_{t}\left[\left(b_{t}-\delta_{t}\right) d t+\sigma_{t} d W_{t}\right], \quad S_{0}=1 .
$$

Here, $W=\left\{\left(W_{t}^{1}, \ldots, W_{t}^{N}\right)^{*} ; t \in \mathbf{T}\right\}$ is a $N$-dimensional Brownian motion on a probability space $(\Omega, F, P)$ and $\left(F_{t}\right)_{t \in \mathbf{T}}$ denotes the $P$-augmentation of the natural filtration generated by $W$. We assume that the sample paths of $W$ specify completely all the distinguishable events, which mathematically entail $F_{T}=F$. Since standard Brownian motions start from zero with probability one, $F_{0}$ is trivial. We will denote by $L_{d}^{2}(\mathbf{T})$ the set of $\left(F_{t}\right)_{t \in \mathbf{T}^{-}}$-progressively measurable processes $\left\{\Psi_{t} ; t \in \mathbf{T}\right\}$ taking values in $R^{d}$ such that

$$
\int_{0}^{T}\left\|\Psi_{t}\right\|^{2} d t<\infty \quad \text { a.s. } P .
$$

Assumption A1 The real-valued interest rate process $\left\{r_{t} ; t \in \mathbf{T}\right\}$, the $N$-dimensional process $\left\{b_{t} ; t \in \mathbf{T}\right\}$, the $N$-dimensional dividend yield process $\left\{\delta_{t} ; t \in \mathbf{T}\right\}$ as well as the volatility $(N \times N)$-matrix-valued process $\left\{\sigma_{t} ; t \in \mathbf{T}\right\}$ are supposed to be progressively measurable with respect to $\left(F_{t}\right)_{t \in \mathbf{T}}$ and bounded uniformly in $(t, \omega)$ in $\mathbf{T} \times \Omega$.

Under this assumption ${ }^{2}$, Equation (2) admits a unique real-valued, $\left(F_{t}\right)_{t \in \mathbf{T}^{-}}$ adapted, continuous solution $\left\{S_{t} ; t \in \mathbf{T}\right\}$, satisfying $\mathrm{E}\left[\sup _{t \in \mathbf{T}} S_{t}^{2}\right]<\infty$.

Assumption A2 For all $t$ in $\mathbf{T}$, the volatility matrix $\sigma_{t}$ has full rank $N$ and the norm of $\left(\sigma_{t}\right)^{-1}$ is uniformly bounded.

Therefore, a $N$-dimensional process $\theta=\left\{\theta_{t} ; t \in \mathbf{T}\right\}$ can be defined by ${ }^{3}$ :

$$
\theta_{t} \equiv\left(\sigma_{t}\right)^{-1}\left[\left(b_{t}-r_{t} 1_{N}\right)\right] \quad P \text { a.s., } \quad 0 \leq t \leq T .
$$

With the above assumptions, $\theta$ is $\left(F_{t}\right)_{t \in \mathbf{T}}$-progressively measurable and uniformly bounded.

We shall also introduce the discounted price process $\tilde{S}=\left\{\tilde{S}_{t} ; t \in \mathbf{T}\right\}$ defined by $\tilde{S}_{t} \equiv S_{t} \exp \int_{0}^{t}\left(\delta_{s}-r_{s}\right) d s$ for all $t$ in $\mathbf{T}$. Using Itô's Lemma, we easily get that $\tilde{S}$ is the unique solution of the following stochastic differential equation:

$$
d \tilde{S}_{t}=\tilde{S}_{t}\left[\left(b_{t}-r_{t}\right) d t+\sigma_{t} d W_{t}\right]=\tilde{S}_{t} \sigma_{t}\left[\theta_{t} d t+d W_{t}\right], \quad \tilde{S}_{0}=1 .
$$

\footnotetext{
${ }^{1}$ All vectors are column vectors and transposition is denoted by the superscript $*$. We denote by $\|Z\|^{2}$ the nonnegative real number $\sum_{i=1}^{n}\left(Z^{i}\right)^{2}$.

${ }^{2}$ See for instance Karatzas-Shreve [1988]

${ }^{3}$ As usual, $1_{d}$ denotes the $d$-dimensional vector whose component are equal to one.
} 
Notice that assets prices can fluctuate in an almost arbitrary, not necessarily Markovian way.

We know that in such a model, there exists a unique equivalent probability measure $\bar{P}$ defined on $(\Omega, F, P)$ that makes the full process $\tilde{S}$ a martingale for $\left(F_{t}\right)_{t \in \mathbf{T}}$. It is given by ${ }^{4}$

$$
d \bar{P} / d P=\mathcal{E}_{T}(-\theta) .
$$

We then have ${ }^{5} d \tilde{S}=\operatorname{diag}\left[\tilde{S}_{t}\right] \sigma_{t} d W_{t}^{\bar{P}}$, where $\left\{W_{t}^{\bar{P}} ; t \in \mathbf{T}\right\}$ is the $\bar{P}$-Brownian motion for $\left(F_{t}\right)_{t \in \mathbf{T}}$ defined by $W_{t}^{\bar{P}} \equiv W_{t}+\int_{0}^{t} \theta_{s} d s$ for all $t$ in $\mathbf{T}$ (see Girsanov's Theorem). We shall denote in the following, the martingale process $\left\{\mathrm{E}\left[\frac{d \bar{P}}{d P} \mid F_{t}\right] ; t \in \mathbf{T}\right\}$ by $\bar{M}=\left\{\bar{M}_{t} ; t \in \mathbf{T}\right\}$.

In the context of the above market-model, consider an agent who starts out with an initial capital $x$ and can decide of the amounts $\pi_{t}=\left(\pi_{t}^{1}, \cdots, \pi_{t}^{N}\right)$ that he invests at time $t$ in the different assets, and of the rate $c_{t}$ at which he withdraws funds for consumption. Assuming that at each time $t$, sales and dividends must finance purchases and consumption, the corresponding wealth process, denoted by $X_{t}^{x ; \pi, c}$, satisfies the following stochastic differential equation

$$
\begin{aligned}
d X_{t}^{x ; \pi, c} & =\sum_{i=0}^{N} \frac{\pi_{t}^{i}}{S_{t}^{i}}\left(d S_{t}^{i}+\delta_{t}^{i} S_{t}^{i} d t\right)-c_{t} d t \\
X_{0}^{x ; \pi, c} & =x
\end{aligned}
$$

which can be rewritten

$$
\begin{aligned}
d X_{t}^{x ; \pi, c} & =\left[r_{t} X_{t}^{\pi, c}-c_{t}\right] d t+\left(\pi_{t}\right)^{*} \sigma_{t} d W_{t}^{\bar{P}} \\
X_{0}^{x ; \pi, c} & =x
\end{aligned}
$$

The set of investment-consumption strategies $(\pi, c)$ satisfying the previous self-financing condition and the following no-bankruptcy condition is called the admissible strategies set and denoted by $\mathbf{A}(x)$ :

$$
\forall t \in \mathbf{T}, X_{t}^{x ; \pi, c} \geq 0, \quad \text { (No-bankruptcy condition) }
$$

This last condition amounts to saying that at each time $t$, the investor must be able to cover his debts -see e.g. Karatzas-Lehoczky-Shreve (1987) or Duffie (1992) where the same assumption is made.

${ }^{4}$ For any $R^{d}$-valued process $\Psi=\left\{\Psi_{t} ; t \in \mathbf{T}\right\}$ in $L_{d}^{2}(\mathbf{T})$, let the real-valued process $\mathcal{E}(\Psi)=$ $\left\{\mathcal{E}_{t}(\Psi) ; t \in \mathbf{T}\right\}$ denote the exponential local martingale given for each $t$ in $\mathbf{T}$ by $\mathcal{E}_{t}(\Psi)=$ $\exp \left\{\int_{0}^{t}\left(\Psi_{s}\right)^{*} d W_{s}-1 / 2 \int_{0}^{t}\left\|\Psi_{s}\right\|^{2} d s\right\} \ldots$

${ }^{5}$ If $Z=\left(Z^{1}, \ldots, Z^{n}\right)$ denotes a vector in $R^{n}$, then $\operatorname{diag} Z$ denotes the $(n \times n)$ diagonal matrix whose diagonal entries are the components of $Z$. 
Under the self-financing condition, the process

$$
Y \equiv\left\{\exp \left(-\int_{0}^{t} r_{s} d s\right) X_{t}^{x ; \pi, c}+\int_{0}^{t} \exp \left(-\int_{0}^{s} r_{u} d u\right) c_{s} d s ; t \in \mathbf{T}\right\}
$$

consisting in the current discounted wealth plus the total discounted consumption is a $\bar{P}$-supermartingale (Fatou's Lemma). It is then easy to see that the market excludes any arbitrage opportunity which turns out to be characterized in our context by the existence of a pair $(\pi, c)$ in $\mathbf{A}(0)$ such that $\left.\mathrm{P}\left[X_{T}^{0 ; \pi, c}>0\right]>0\right)$.

Let $\mathbf{B}$ denote the set of pairs $(X, c)$ where $c$ is an adapted nonnegative consumption rate process and $X$ is a nonnegative $F_{T}$-measurable random variable describing the terminal wealth. An agent is represented by a utility function $U: \mathbf{B} \rightarrow \mathbb{R}$, given by

$$
U(c, X)=\mathrm{E}\left[\int_{0}^{T} u\left(t, c_{t}\right) d t+V(X)\right],
$$

where $u$ and $V$ satisfy the following assumption.

Assumption $\mathcal{U}$ The function $V: R_{+} \rightarrow R i s^{6} C^{1}$ on $(0, \infty)$, strictly increasing, strictly concave and satisfies Inada conditions (i.e., $\inf _{x} V_{j}^{\prime}(x)=0$ and $\left.\sup _{x} V_{j}^{\prime}(x)=+\infty\right)$. The function $u_{j}: \mathbf{T} \times R_{+} \rightarrow R$ is $C^{0,1}$ on $\mathbf{T} \times R_{+}^{*}$, strictly increasing, strictly concave and satisfies Inada conditions.

Under Assumption $\mathcal{U}$, we shall denote by $u_{c}(t, \cdot)$ the derivative of $u(t, \cdot)$ and by $I_{u}(t, \cdot)$ the inverse function of $u_{c}(t, \cdot)$, which is a strictly decreasing continuous function on $(0, \infty)$ in $(0, \infty)$. We shall also denote by $I_{V}$ the inverse function of $V^{\prime}(\cdot)$.

The considered agent has an initial endowment $x$ and tries to maximize his utility $U(c, X)$ on both his consumption over the time-interval $\mathbf{T}$ and his terminal wealth. The optimal demand $(c)^{*}$ of the agent in the consumption commodity as well as his optimal portfolio $(\pi)^{*}$ are determined by the optimization problem

$$
\sup _{(\pi, c) \in \mathbf{A}(x)} U\left(c, X_{T}^{x_{j} ; \pi, c}\right)=\sup _{(X, c) \in \mathbf{B}} U(c, X) .
$$

Adapting the proofs of Duffie (1994) and Karatzas (1989), we get that

Proposition 2 A pair $\left(c^{*}, X^{*}\right)$ in $\mathbf{B}$ is optimal for an agent with an initial endowment $x$ if and only if there exists a constant $\gamma^{*}>0$ such that

$$
\begin{aligned}
c^{*}(t) & =I_{u}\left(t, \frac{1}{\gamma^{*}} \exp \left(-\int_{0}^{t} r_{s} d s\right) \bar{M}_{t}\right) \quad 0 \leq t \leq T \quad \text { a.s. } P \\
X^{*} & =I_{V}\left(\exp \left(-\int_{0}^{T} r_{s} d s\right) \bar{M}_{T}\right)
\end{aligned}
$$

\footnotetext{
${ }^{6}$ As usual, a function $F: \mathbf{T} \times R \rightarrow R$ is said to be of class $C^{m, n}$ if the $m$-th derivative of $F(\cdot, x): \mathbf{T} \rightarrow R$ and the $n$-th derivative of $F(t, \cdot): R \rightarrow R$ exist and are continuous.
} 


$$
\mathrm{E}\left[\int_{0}^{T} \exp \left(-\int_{0}^{t} r_{s} d s\right) \bar{M}_{t} c^{*}(t) d t+\exp \left(-\int_{0}^{T} r_{s} d s\right) \bar{M}_{T} X^{*}\right]=x
$$

This last proposition permits to solve explicitly the agent's optimization program. Huang and Pagès (1992) extended this methodology to the infinite horizon framework. Karatzas, Lehoczky, Sethi and Shreve (1986) provided explicit computations in that framework assuming constant coefficients in the price evolution equations. He and Pearson (1991 a and b) and Karatzas, Lehoczky, Shreve and $\mathrm{Xu}$ (1991) extended the methodology to incomplete markets and proved that the optimal investment/consumption plan is given, as in the classical case, by the inverse of the marginal utility evaluated at the random variable which is optimal for a well-defined dual problem. Cvitanic̀ and Karatzas (1992) used the same approach in order to solve the problem when there is convex constraints on the strategies (short-sales constraints, borrowing constraints,...). Fleming and Zariphopoulou (1991) solved the problem assuming different borrowing and lending rates. Cuoco (1997) and El Karoui and Jeanblanc-Picqué (1997) considered random endowment streams. Cvitanic̀ and Ma (1996) and Cuoco and Cvitanic (1998) generalized these results in the context of a "large investor". In that context, the strategy of the investor has a direct nonlinear impact on the price dynamics. The main technique in all these references consists in embedding the original problem into a family of perfect (linear) "fictitious" markets, where security prices dynamics are modified and agents receive an additional stochastic "endowment" reflecting the nonlinearity in the market price of risk. The fictitious markets are designed in such a way that the optimal policy in one of them coincides with that in the actual, nonlinear market.

Using the partial differential equations (PDE) approach, Dumas and Luciano (1989) first formulated the problem in the presence of transaction costs. The main contributions in this context are Davis and Norman (1990), Fleming, Grossman, Vila and Zariphopoulou (1990) and Shreve and Soner (1994).

When there are imperfections, the utility maximization approach can be used in order to provide pricing formulas for new contingent claims. There are mainly two methods. The first one, initiated by Hodges and Neuberger (1989) in the transaction costs setting, consists in using the marginal utility of the considered agent at his optimal consumption-investment plan as a state-price density. The second method, initiated by Davis (1994) consists in comparing the optimal utility levels $\mathbb{V}^{x}$ with a deterministic initial endowment $x$ and $\mathbb{V}^{*}$ with a stochastic endowment equal to the payoff of the considered claim. The "fair" price $x$ is then defined by the equation

$$
\mathbb{V}^{x}=\mathbb{V}^{*}
$$

Papers along these lines include Constantinides (1986), Panas (1993), Davis, Panas and Zariphopoulou (1993), Davis and Panas (1994), Davis and Zariphopoulou (1995), Cvitanic and Karatzas (1996), Constantinides and Zariphopoulou (1997) and Barles and Soner (1998). 
In this issue, Cvitanic̀ and Wang (2001) show that the martingale/duality approach adopted in the frictionless model works also in the transaction costs framework and prove that the optimal terminal wealth is given as the inverse of marginal utility evaluated at the random variable which is optimal for an appropriately defined dual problem. They prove the existence of a solution for this dual problem and doing so they resolve a question left open by Cvitanic and Karatzas (1996). A similar problem is studied by Deelstra, Pham and Touzi (2000) where the utility functions are defined on $\mathbb{R}^{d}$ instead of $\mathbb{R}$.

Framstad, Øksendal and Sulem (2001) considers also the transaction costs framework but in a jump diffusion market. Using a viscosity solution approach, they show that the solution of the problem in that context has the same form as in the pure diffusion case : there is a no-transaction cone such that it is optimal to make no transactions as long as the wealth position remains in that cone and to trade on the boundary. Bellamy (2001) solves the same problem but assuming market incompleteness instead of the presence of transaction costs and using a Hamilton-Jacobi-Bellman (HJB) approach.

Using filtering techniques, Lakner (1995) considers utility maximization problems where the agent must estimate the mean rate of return of the assets. In this issue, Dokuchaev and Zhou (2001) considers the case where the stock appreciation rates are not observable and where the strategies depend only on the known distribution of these rates and on the current prices. Furthermore, they use general utility/loss functions (including mean-variance criteria and goal achieving problems) and they consider some lower and upper constraints on the terminal wealth. The problem is solved by means of backward stochastic differential equations as well as a dual formulation.

Finally, we want to mention another family of optimization problems related to the contingent claims pricing : the hedging problems. These problems are not represented in this special issue but are studied by many papers in the recent literature. The main problem in all these paper is to compute the hedging price of a given contingent claim with respect to a given hedging criterion. If we assume that the agents want to minimize the downside risk, then the hedging price is equal to the super-replication price and its computation leads to solve a stochastic-control-based problem as in the pionneering paper of El Karoui and Quenez $(1991,1995)$. If we assume that the agents want to minimize the quadratic risk then we have to solve the mean-variance hedging problem and we refer to Föllmer and Schweizer (1991) or Schweizer (1993) for a survey about related results.

\section{The equilibrium}

Models of competitive equilibrium go back to Walras (1874). The first complete proof for the existence of an equilibrium in an economy with finitely many commodities was given by Arrow and Debreu (1954). In the chapter 7 of Debreu (1959), the author explains how this model permits to take into account dynamic markets with uncertainty. Bewley (1972) studied the competitive equi- 
librium in an infinite-dimensional commodity space, namely $L^{\infty}$ and Mas-Colell (1986) generalized Bewley's results to Hausdorff locally convex, topological vector spaces under a "uniform properness condition" on the agent's preferences. Araujo and Monteiro (1989 a and b) and Duffie and Zame (1989) proved independently the existence of an equilibrium without Mas-Colell's uniform properness condition. Dana, Le Van and Magnien (1997) extended Mas Colell's result to topological locally solid Riesz spaces under a local non-satiation condition weaker than the uniform properness one. Aase (1992) and Bernis (2000) applied these results to the reinsurance markets.

All the previous models does not take explicitely into account dynamic security trading. Models where the agents achieve equilibrium allocations by trading in securities like the capital asset pricing model $(\mathrm{CAPM})$ or the consumption based capital asset pricing model (CCAPM) can be found in the literature going back to Merton (1971), Cox, Ingersoll and Ross (1985), Duffie and Huang (1985), Huang (1987) and Karatzas, Lehoczky and Shreve (1990).

The link between these two approaches is made by Duffie and Huang (1985) where the authors explain how an Arrow-Debreu equilibrium can be implemented by trading in securities. This role of securities was, in fact, already recognized by Arrow (1952). The difference between the two approaches is illustrated by Cuoco (1997) where the budget constraints are associated to all the possible equilibrium prices (all the risk-neutral measures) instead of a unique budget constraint associated to the equilibrium price as in the classical general equilibrium model.

In Karatzas, Lehoczky and Shreve (1990), all agents are endowed in units of the same perishable commodity, which arrives at some time-varying random rate. Agents may consume their endowment as it arrives, they may sell some portion of it to other agents, or they may buy extra endowment from other agents. The endowment, however, cannot be stored, and agents wish to hedge the variability in their endowment process by trading with one another.

In this model all the prices are in term of a unique consumption good. When the market is complete it is equivalent to assume that the agents receive their endowment initially rather than over time. In that case and in order to have a stochastic total wealth, we assume that the consumption good is produced by the firms and distributed as dividends among the shareholders. The equilibrium condition imposes then a total consumption equal to the total supply of the consumption good and a total investment in each firm equal to the total value in term of consumption good of that firm.

With the notations of Jouini and Napp (1998), the mathematical description of the model is the following. Let $\mathbb{E}$ be an economy with $n$ agents indexed by $j=1, \cdots, n$ and let us assume that the $j^{t h}$ agent has an initial wealth $x_{j}$ and a utility function $U_{j}: \mathbf{B} \rightarrow \mathbb{R}$ given by

$$
U_{j}(c, X)=\mathrm{E}\left[\int_{0}^{T} u_{j}\left(t, c_{t}\right) d t+V_{j}(X)\right]
$$

where $u_{j}$ and $V_{j}$ satisfy our assumption $\mathcal{U}$. As previously each agent maximizes 
his utility level over the set of admissible strategies. More precisely, the $j^{\text {th }}$ agent problem is

$$
\sup _{(\pi, c) \in \mathbf{A}\left(x_{j}\right)} U_{j}\left(c, X_{T}^{x_{j} ; \pi, c}\right) .
$$

In this framework an equilibrium consists in a $N$-dimensional price processes $S$ and trading-consumption choices $\left(\pi_{j}^{*} ; c_{j}^{*}\right)_{1 \leq j \leq n}$ which are optimal for the agents, i.e.

$$
\left(\pi_{j}^{*} ; c_{j}^{*}\right) \in \arg \max _{(\pi, c) \in \mathbf{A}\left(x_{j}\right)} U_{j}\left(c, X_{T}^{x_{j} ; \pi, c}\right)
$$

and such that for all $t$ in $\mathbf{T}$, the following market clearing conditions hold almost surely:

$$
\begin{aligned}
\sum_{j=1}^{n}\left(c_{j}^{*}\right)_{t} & =\delta_{t} \cdot 1_{N} \\
\sum_{j=1}^{n}\left(\pi_{j}^{*}\right)_{t}^{i} & =q^{i} S_{t}^{i}, \quad i=0, \cdots, N \\
\sum_{j=1}^{n} X_{t}^{\pi_{j}^{*}, c_{j}^{*}} & =S_{t} .
\end{aligned}
$$

where $q^{i}$ is the number of firm $i$ outstanding shares

Note that the last condition is redundant with the two previous ones by the self-financing condition.

In Karatzas, Lehoczky and Shreve (1990) it is shown that under mild conditions a unique equilibrium exists. In this issue, Chiarolla and Haussmann (2001) specializes and extends the Karatzas and al. (1990) model to a situation where the endowment streams of the agents are denominated in money, not in goods, and are not exogenous. The labor provided by the agents to a firm produces the consumable good through a production function. The agents have then to choose a consumption and a leisure levels in order to maximize their utility function. Furthermore, the firm defines the level of employment by a profit maximization program. The utility functions of the agents depend then on two control variables and the main contribution of this paper is to extend the classical one-dimensional approach to this framework. The authors provide first order necessary conditions for equilibrium, and derive from there the existence of such an equilibrium. They also solve explicitly two examples.

Basak and Croitoru (2001) exploit the equilibrium conditions in order to analyze the taxation impact on the asset prices. They consider a simple two agents model and use the "fictitious" market techniques described in the previous section in order to solve the individual utility maximization problem. The main difficulty is due to the presence of two redundant assets but with different taxation rules. The redundancy adds an extra step in the agent's problem : once he has chosen his risk exposure, he must decide how to allocate that risk 
between the two securities. The authors establish general necessary conditions for equilibrium and show, in particular, that arbitrage opportunities still exist at the optimum. They characterize this "mispricing" and they provide an analysis of its equilibrium role.

\section{Acknowledgements}

Some of the papers gathered here have been presented at the International Conference on Mathematical Finance, Hammamet, Tunisia, 14-18 juin 1999 organized by Nizar Touzi and the author. We wish to thank all the participants at this conference for helpful discussions as well as the members of the scientific committee (Gérard Debreu (President), Freddy Delbaen, Ivar Ekeland, Ioannis Karatzas, Pierre-Louis Lions, Stanley Pliska and Dieter Sondermann), the members of the organizing committee (Guillaume Bernis, Abdelhamid Bizid, Laurence Carassus, Kaïs Hamza, Clotilde Napp, Abdelhamid Trad and Amel Zenaïdi) and our sponsors (UNESCO, Tunisian authorities, French authorities, ADRES, Tunisie-Valeurs group, Amen Invest and the Tunis Stock Market). We are also most grateful to the Journal of Mathematical Economics and to its Editor, Bernard Cornet, for welcoming us in print. I have also benefitted from comments and discussions with Guillaume Bernis, Abdelhamid Bizid, Bruno Bouchard, Martino Grasselli, Clotilde Napp and Nizar Touzi in the writing of this presentation. I am also indebted to CREST for the very stimulating scientific atmosphere during the 1997-2000 period and to the Stern Business School where this paper was written for inviting me during the 1999-2000 period.

\section{References}

[1] Aase K. (1992), Dynamic equilibrium and the structure premium in a reinsurance market. Geneva Papers on Risk and Insurance 17, 93-136.

[2] Ansel, J.P. and C. Stricker (1992), Lois de martingale, densités et décomposition de Föllmer-Schweizer, Ann. Inst. Henri Poincaré 28, 375-392,

[3] Ansel, J.P. and C. Stricker (1993), Unicité et existence de la loi minimale, Séminaire de Probabilités, XXVII, Lect. Notes Math, 1557, SpringerVerlag, 22-29

[4] Ansel, J.P. and C. Stricker (1994), Couverture des actifs contingents, Ann. Inst. Henri Poincaré 30, 303-315,

[5] Araujo, A. and P. K. Monteiro (1989a), Equilibrium without uniform conditions. Journal of Economic Theory 48, 416-427,

[6] Araujo, A. and P. K. Monteiro (1989b), General equilibrium with infinitely many goods : The case of separable utilities. In Equilibrium and dynamics : Essays in honor of David Gale. McMillan, London. 
[7] Arrow, K. (1952), Le rôle des valeurs boursières pour la répartition la meilleure des risques, Econométrie, Colloque International du CNRS, Paris 40, 41-47 (with discussion 47-48). English translation in Review of Economic Studies 31 (1964), 1-96.

[8] Arrow, K. and G. Debreu (1954), Existence of equilibrium for a competitive economy. Econometrica 22, 265-290.

[9] Barles G. and H. M. Soner (1998), Option pricing with transaction costs, and a nonlinear Black-Scholes equation, Finance and Stochastics 2(4).

[10] Basak S. and B. Croitoru (2001), Nonlinera taxation, tax arbitrage and equilibrium asset prices, Journal of Mathematical Economics, this issue.

[11] Bellamy N. (2001), Wealth optimization in an incomplete market driven by a jump-diffusion process. Journal of Mathematical Economics, this issue.

[12] Bernis (2000), Equilibrium in a reinsurance market with short sale constraints. Preprint. Université de Paris I Panthéon-Sorbonne.

[13] Bewley, T. (1972), Existence of equilibria in economies with infinitely many commodities. Journal of Economic Theory 4, 514-540.

[14] Black, F. and M. Scholes (1973), The pricing of options and corporate liabilities, Journal of Political Economy 81, 637-659.

[15] Carassus L. and E. Jouini (1997), Coûts de transaction et contraintes de vente à découvert : une approche unifiée. DP CREST n ${ }^{\circ} 9758$.

[16] Carassus L. and E. Jouini (1998), Investment and arbitrage opportunities with short sales constraints. Mathematical Finance 8(3), 169-178.

[17] Carassus L. and E. Jouini (2000), A discrete stochastic model for investment with an application to the transaction costs case. Journal of Mathematical Economics 33(1), 57-80.

[18] Chiarolla M.B.and U.G. Haussmann (2001), Equilibrium in a stochastic model with consumption, wages and investment. Journal of Mathematical Economics, this issue.

[19] Constantinides G.M. (1986), Capital market equilibrium with transaction costs, Journal of Political Economy 94, 842-862.

[20] Constantinides G.M.and Th. Zariphopoulou (1997), Bounds on option prices in an intertemporal economy with proportional transaction costs and general preferences. Preprint.

[21] Cox, J.C. and C.F. Huang (1989), Optimal consumption and portfolio policies when asset prices follow a diffusion process. Journal of Economic Theory 49, 33-83. 
[22] Cox, J.C. and C.F. Huang (1991), A variational problem arising in financial economics. Journal of Mathematical Economics 20, 465-487.

[23] Cox, J.C., Ingersoll, J.E. and S. Ross (1985), An intertemporal general equilibrium model of asset prices, Econometrica 53, 363-384.

[24] Cox, J.C. and S. Ross (1976), The valuation of options for alternative stochastic processes, Journal of Financial Economics 3, 145-166.

[25] Cuoco, D. (1997), Optimal consumption and equilibrium prices with portfolio constraints and stochastic income. Journal of Economic Theory 72, 33-73.

[26] Cuoco, D. and J. Cvitanic̀ (1998), Optimal consumption choices for a "large investor". Journal of Economic Dynamics and Control 22, 401-436.

[27] Cvitanic̀, J. and I. Karatzas (1992), Convex duality in constrained portfolio optimization. Annals of Applied Probability 2, 767-818.

[28] Cvitanic̀, J. and I. Karatzas (1996), Hedging and portfolio optimization under transaction costs : a martingale approach. Mathematical Finance $6,133-165$.

[29] Cvitanic̀, J. and J. Ma (1996), Hedging options for a large investor and forward-backward SDEs. Annals of Applied Probability 6, 370-398.

[30] Cvitanic̀, J., Pham, H. and N. Touzi (1999), A closed form solution to problems of super-replication under transaction costs. Finance and stochastics 3 (1), 35-54.

[31] Cvitanic̀, J. and H. Wang (2001), On optima terminal wealth under transaction costs. Journal of Mathematical Economics, this issue.

[32] Dalang, R.C., Morton, A. and W. Willinger (1990), Equivalent martingale measures and no-arbitrage in stochastic security market models. Stochastics 29, 185-201.

[33] Dana R.-A., Le Van C. and F. Magnien (1997), General equilibrium in asset markets with or without short-selling. Journal of Mathematical Analysis and Applications 206, 567-588.

[34] Davis M.H.A. (1994), Option pricing in incomplete markets. Preprint, Imperial College, London.

[35] Davis M.H.A. and A. Norman (1990), Portfolio selection with transaction costs. Mathematics of Operations Research 15, 676-713.

[36] Davis, M.H.A. and V.G. Panas, (1994), The writting price of a European contingent claim under proportional transaction costs. Comp. \& App. Mathematics 13, 155-117. 
[37] Davis, M.H.A., Panas, V.G., and T. Zariphopoulou, (1993), European option pricing with transaction costs. SIAM J. Control \& Optimization $31,470-493$.

[38] Davis, M.H.A. and T. Zariphopoulou, (1995), American options and transaction fees. Mathematical Finance (M. Davis, D. Duffie, W. Fleming and S. Shreve, eds.) Ima Vol. 65, 47-62. Springer-Verlag, New-York.

[39] Debreu, G. (1959), Theory of Value. J. Wiley \& Sons, New-York.

[40] Deelstra G., Pham H. and N. Touzi (2000), Dual formulation of the utility maximization problem under transaction costs. Preprint. CREST.

[41] Delbaen, F. (1992), Representing martingale measures when asset prices are continuous and bounded, Mathematical Finance 2 (2), 107-130.

[42] Delbaen, F. and W. Schachermayer (1994), A general version of the fundamental theorem of asset pricing, Matematische Annalen 300, 463-520.

[43] Dokuchaev N. and X.Y. Zhou (2001), Optimal investment strategies with bounded risks, general utilities and goal achieving. Journal of Mathematical Economics, this issue.

[44] Duffie D, (1992), Dynamic asset pricing theory. Princeton University Press.

[45] Duffie D. and C.F. Huang, (1985), Implementing Arrow-Debreu equilibria by continuous of few long-lived securities. Econometrica 53, 1337-1356.

[46] Duffie, D., and C.F. Huang, (1986), Multiperiod security market with differential information, Journal of Mathematical Economics, 15, p. 283303.

[47] Duffie D. and W. Zame, (1989), The consumption-based capital asset pricing model. Econometrica 57, 1279-1297.

[48] Dumas, B. and E. Luciano, (1989), An exact solution to a dynamic portfolio choice problem under transaction costs. J. Finance 46, 577-595.

[49] El Karoui, N. and M. Jeanblanc-Picqué (1998), Optimization of consumption with labor income. Finance and Stochastics 2(4).

[50] El Karoui, N. and M.C. Quenez, (1991), Progammation dynamique et évaluation des actifs contingents en marché incomplet. C. R. Acad. Sci, Paris, Série I 313, 851-854.

[51] El Karoui, N. and M.C. Quenez, (1995), Dynamic programming and pricing of contingent claims in an incomplete market. SIAM J. control $\mathscr{G}$ Optimization 33, 29-66. 
[52] Fleming, W., Grossman, S., Vila, J.L., and T. Zariphopoulou, (1990), Optimal portfolio rebalancing with transaction costs. Preprint, Division of Applied Math., Brown University.

[53] Fleming, W. and T. Zariphopoulou, (1991), An optimal investment consumption model with borrowing. Math. Operation Research 16, 802-822.

[54] Föllmer, H. and M. Schweizer, M. (1991) : Hedging of contingent claims under incomplete information., Applied Stochastic Analysis, Stochastic Monographs vol.5, Gordon and Breach.

[55] Framstad N.C., Øksendal B. and A. Sulem (2001), Optimal consumption and portfolio in a jump diffusion market with proportional transaction costs. Journal of Mathematical Economics, this issue.

[56] Harrison, J., and D. Kreps (1979), Martingales and arbitrage in multiperiod securities markets. Journal of Economic Theory 20, 381-408.

[57] Harrison, J., and S. Pliska (1981), Martingales and stochastic integrals in the theory of continuous trading. Stochastic Processes and their Applications 11, 215-60.

[58] Harrison, J., and S. Pliska (1983), A stochastic calculus model of continuous trading : complete markets, Stochastic Processes \& Appl. 15, 313-316.

[59] He, H. and N. Pearson, (1991a), Consumption and portfolio politicies with incomplete markets and short-sale constraints : the infinite-dimensional case. J. Economic Theory 54, 259-304.

[60] He, H. and N. Pearson, (1991b) Consumption and portfolio politicies with incomplete markets and short-sale constraints : the finite-dimensional case. Mathematical Finance 1 (3), 1-10.

[61] Hodges, S.D. and A. Neuberger, (1989), Optimal replication of contingent claims under transaction costs. Rev. Futures Markets 8, 222-239.

[62] Huang, C.F. (1987), An intertemporal general equilibrium asset pricing model : the case of diffusion information. Econometrica 55, 117-142.

[63] Huang, C.F. and H. Pagès, (1992), Optimal consumption and portfolio policies with an infinite horizon : existence and convergence. Ann. Appl. Probability 2, 36-64.

[64] Jouini, E. (1997) Market imperfections, equilibrium and arbitrage. In Financial mathematics, W.J. Runggaldier (Ed), Lectures Notes in Mathematics series 1656, Springer Verlag, Berlin.

[65] Jouini, E., and H. Kallal (1995a), Martingales and arbitrage in securities markets with transaction costs. Journal of Economic Theory 66 (1), 178197. 
[66] Jouini, E., and H. Kallal (1995b), Arbitrage in securities markets with short sales constraints. Mathematical Finance 5(3), 197-232

[67] Jouini, E. and H. Kallal (1999), Viability and equilibrium in securities markets with frictions. Mathematical Finance 9(3), 275-292.

[68] Jouini E., Kallal H. and C. Napp (2001), Arbitrage in financial markets with fixed costs, Journal of Mathematical Economics, this issue.

[69] Jouini, E. and C. Napp (1998), Continuous time equilibrium pricing of nonredundant assets. DP CREST $n^{\circ} 9830$.

[70] Jouini E. and C. Napp (2000), Arbitrage and investment opportunities, to appear in Stochastics and Finance,

[71] Jouini E., Napp C. and W. Schachermayer (2000), A remark on Kreps-Yan theorem, Preprint.

[72] Kabanov, Yu. (1999), Hedging and liquidation under transaction costs in currency markets. Finance and Stochastics 3, 237-248.

[73] Kabanov, Yu. and D.O. Kramkov, (1994 a), No-arbitrage and equivalent martingale measures : an equivalent proof of the Harrison-Pliska theorem. Theory Probab. Appl. 39, 523-527.

[74] Kabanov, Yu. and D.O. Kramkov, (1994 b), Large financial markets : asymptotic arbitrage and contiguity. Theory Probab. App. 39, 222-229.

[75] Kabanov Yu. and Ch. Stricker (2001), The Harrison-Pliska arbitrage pricing theorem under transaction costs. Journal of Mathematical Economics, this issue.

[76] Karatzas, I. (1989), Optimization problems in the theory of continuous trading. SIAM J. Control \& Optimization 27, 1221-1259.

[77] Karatzas, I., Lehoczky, J.P., Sethi, S.P., and S.E. Shreve, (1986), Explicit solution of a general consumption/investment problem. Math. Operations Research 11, 261-294.

[78] Karatzas, I., Lehoczky, J.P., and S.E. Shreve,(1987), Optimal portfolio and consumption decisions for a "small investor" on a finite horizon. SIAM J. Control \& Optimization 25, 1557-1586.

[79] Karatzas, I., Lehoczky, J.P., and S.E. Shreve, (1990), Existence and uniqueness of multi-agent equilibrium in a stochastic, dynamic consumption/investment model. Math. Operations Research 15, 80-128.

[80] Karatzas, I., Lehoczky, J.P., Shreve, S.E., and G.L. Xu, (1991), Martingale and duality methods for utility maximization in an incomplete market. SIAM J. Control \& Optimization 29, 702-730. 
[81] Kramkov, D. and W. Schachermayer, (1997), The asymptotic elasticity of utility functions and optimal investment in incomplete markets. Preprint. University of Wien.

[82] Kreps, D. (1981), Arbitrage and equilibrium in economies with infinitelymany commodities. J. Math. Economics 8, 15-35.

[83] Kusuoka, S. (1995), Limit theorem on option replication with transaction costs. Annals of applied probability 5, 198-221.

[84] Lakner, P. (1993), Martingale memasures for a class of right-continuous processes. Mathematical Finance 3 (1), 43-53.

[85] Lakner, P. (1995), Utility maximization with partial information. Stochastic Processes and their Applications 56, 247-273.

[86] Mas-Colell, A. (1986), The price equilibrium existence problem in topological vector lattices. Econometrica 54, 1039-1053.

[87] Merton, R.C. (1969), Lifetime portfolio selection under uncertainty : the continuous-time case. Rev. Econom. Statist. 51, 247-257.

[88] Merton, R.C. (1971), Optimum consumption and portfolio rules in a continuous-time model. J. Econom. Theory 3, 373-413. Erratum : ibid. 6 (1973), 213-214.

[89] Napp (2001), to appear in the Journal of Mathematical Economics.

[90] Panas, V.G. (1993), Option pricing with transaction costs. Doctoral dissertation, Imperial College, London.

[91] Pliska, S.R. (1986), A stochastic calculus model of continuous trading : optimal portfolios, Math. Operations Research 11, 371-382.

[92] Rogers, L.C.G. (1995), Equivalent martingale measures and no-arbitrage. Stochastics 51, 41-50.

[93] Schachermayer, W. (1992), A Hilbert-space proof of the fundamental theorem of asset-pricing in finite discrete time. Insurance : Mathematics $\&$ Economics 11, 249-257.

[94] Schachermayer, W. (1993), A couterexample to serveral problems in mathematical finance. Mathematical Finance 3 (2), 217-229.

[95] Schachermayer, W. (1994), Martingale measures for discrete-time processes with infinite horizon,. Mathematical Finance 4 (1), 25-56.

[96] Schweizer, M. (1992b), Mean-variance hedging for general claims. Annals Appl. Probability 2, 11-179.

[97] Shirakawa, H. and H. Konno (1995), "Pricing of options under the proportional transaction costs," preprint, Tokyo Institute of Technology. 
[98] Shreve, S.E. and H.M. Soner, (1994), Optimal investment and consumption with transaction costs. Ann. Appl. Probab. 4, 609-692.

[99] Stricker, C. (1990), Arbitrage et lois de martingales. Ann. Inst. HenriPoincaré 26, 451-460.

[100] Walras, L. (1874), Eléments d'économie politique pure. Fourth edition, L. Corbaz, Lausanne. English translation in Elements of Pure Economics (W. Jeffé, ed.) Allen \& Unwin, London. 